\title{
Corela
}

Cognition, représentation, langage

HS-19 | 2016

Le point de vue pris au mot

\section{La relation entre la description topique des mots et la dimension figurative des proverbes. De la sémiotique à la Sémantique des Points de Vue}

\section{Marzieh Athari Nikazm}

\section{OpenEdition}

Journals

Édition électronique

URL : http://journals.openedition.org/corela/4293

DOI : $10.4000 /$ corela.4293

ISSN : 1638-573X

Éditeur

Cercle linguistique du Centre et de l'Ouest - CerLICO

Référence électronique

Marzieh Athari Nikazm, « La relation entre la description topique des mots et la dimension figurative des proverbes. De la sémiotique à la Sémantique des Points de Vue », Corela [En ligne], HS-19 | 2016, mis en ligne le 08 juin 2016, consulté le 01 mai 2019. URL : http://journals.openedition.org/ corela/4293 ; DOI : 10.4000/corela.4293

Ce document a été généré automatiquement le 1 mai 2019.

\section{(c) (1) (-)}

Corela - cognition, représentation, langage est mis à disposition selon les termes de la licence Creative Commons Attribution - Pas d'Utilisation Commerciale - Partage dans les Mêmes Conditions 4.0 International. 


\title{
La relation entre la description topique des mots et la dimension figurative des proverbes. De la sémiotique à la Sémantique des Points de Vue
}

\author{
Marzieh Athari Nikazm
}

\section{Introduction}

1 Parmi les formulations sentencieuses, les proverbes aussi bien que les maximes et les dictons sont fondés sur des représentations collectives, et de ce fait, font l'objet de différentes disciplines comme l'anthropologie, l'ethnolinguistique, la linguistique, etc. Dans certaines communautés linguistiques, les proverbes constituent le pilier et l'ancrage pour tout propos dans la mesure où ils peuvent s'appliquer dans des situations diversifiées. Comme le dit un proverbe russe: «Sans angles, pas de maison; sans proverbes, pas de paroles".

Dans de nombreuses études, l'analyse du fonctionnement des proverbes s'intègre dans le cadre de la problématique fondamentale que constitue l'intercompréhension ou l'intelligibilité collective. Pour le cadre de notre analyse de quelques proverbes, nous allons recourir à la Sémantique des Points de Vue et à la sémiotique tout en nous intéressant à l'examen des points de vue figés du discours proverbial, qui correspond à la « dimension figurative » du discours.

3 Pour définir les structures sémiotiques (discours figuratif) de l'énoncé proverbial, il est nécessaire de mettre en évidence, d'une part, l'organisation interne et les conditions de sa valeur lexicale et, d'autre part, le fait que les proverbes, par leur scénographie et leur figurativité donnent une image, tout en imposant «une façon de voir» et que cette 
manière de voir est lexicalisée dans les mots, on peut les décrire de manière adéquate en termes de champs topiques lexicaux définis par Pierre-Yves Raccah.

Nous tenterons donc de montrer que les points de vue lexicalisés dans les mots peuvent contribuer à définir la dimension figurative des proverbes. Signalons que ce travail ne pourrait être qu'une piste de recherche pour nos travaux ultérieurs adoptant les principes de cette approche.

\section{La figurativité du discours proverbial}

5 Les proverbes ont une valeur culturelle et ils nous montrent les façons de voir et les manières de penser d'une société donnée. Les théoriciens ont l'habitude d'étudier les proverbes comme des indices révélateurs d'un système social et culturel. Ils parlent en général d'un système de particularités expressives liées aux conditions sociales dans lesquelles la langue est actualisée. Georges Kleiber considère le proverbe comme une "dénomination» dont le sens ne peut pas être calculé à partir des unités qui le composent : il est « donné par avance » (Kleiber 1999: 66). Le sens du proverbe est « fixé par convention pour tout locuteur » (Kleiber $1994: 210$ ). Ainsi ne peut-on pas utiliser un proverbe sans connaître son sens. Il note également que: «L'utilisation d'un proverbe présuppose tout comme celle d'un nom commun la connaissance de son sens ou interprétation standard »(Kleiber 1994 : 211).

D'autres théoriciens comme Yves-Marie Visetti et Pierre Cadiot identifient quatre phases dans les proverbes dont la première est la scénographie qui correspond à la "présence sensible d'une couche figurative» (Visetti et Cadiot 2006: 83-96). Quand nous lisons ou nous entendons un proverbe, nous entrons immédiatement dans la figurativité, à savoir une image du monde se dessine. Cependant la figurativité est définie par des sémioticiens par rapport à la mimesis. Denis Bertrand écrit à ce propos :

Elle suggère spontanément la ressemblance, la représentation, l'imitation du monde par la disposition des formes sur une surface. [...] le concept sémiotique de la figurativité a été étendu à tous les langages, verbaux comme non verbaux, pour désigner cette propriété qu'ils ont en commun de produire et de restituer partiellement des significations analogues à celles de nos expériences perceptives les plus concrètes. La figurativité permet ainsi de localiser dans le discours cet effet de sens particulier qui consiste à rendre sensible la réalité sensible: une de ses formes est la mimesis. (Bertrand 2000 : 97)

7 Le concept de la figurativité peut être illustré par les proverbes qui donnent une image du monde en dessinant l'espace, les objets et les valeurs. Il ne suffit pas de dire qu'on fait voir ou on annonce un «déjà-vu». En effet, on persuade l'interlocuteur par un raisonnement abstrait: «on fait aussi croire». Comme le note Bertrand: «les textes figuratifs sollicitent une forme de rationalité particulière qui est d'ordre analogique et non pas déductif» (Bertrand 2000: 98). Et puisque le proverbe a une structure analogique, sa fonction est d'« exciter la sagacité du destinataire en s'adressant à son imagination: le contenu manifesté est simplement mis en parallèle avec un contexte situationnel analogue, dont l'auditeur est censé tirer la leçon ». (Rodegem $1984: 123$ ). Les sémioticiens parlent aussi de "pensée figurative », de "raisonnement figuratif » et ils évoquent la "profondeur" du figuratif. Elle est placée dans l'énoncé proverbial à la surface. À part la définition apportée ci-dessus, nous en trouvons encore cinq propositions à propos de la figurativité. Si nous n'en avons sélectionné que deux, c'est parce qu'elles sont en relation étroite avec notre étude. Premièrement : 
Le qualificatif figuratif est [...] employé à propos d'un contenu donné (d'une langue naturelle par exemple), quand celui-ci a un correspondant au niveau de l'expression de la sémiotique naturelle (ou du monde naturel). En ce sens, dans le cadre du parcours génératif, la sémantique discursive inclut, avec la composante thématique (ou abstraite), une composante figurative. (Greimas et Courtés 1986 : 146)

Cette définition met en évidence le rapport entre le plan de l'expression et le plan du contenu. Pour chaque forme dans le monde naturel, il y a un contenu dans la langue humaine qui nous est familier car nous avons déjà cette expérience en contactant le monde. Quant aux proverbes, ils nous donnent une image du monde naturel, qui est très facile à comprendre mais il y a un contenu spécifique dans la langue qui est en rapport avec la composante abstraite. Cette composante avec la forme donnée nous présente la composante figurative. Il y a une autre définition proposée par Bertrand :

La figurativité se définit comme tout contenu d'un système de représentation, verbal, visuel, auditif, ou mixte, qui se trouve corrélé à une figure signifiante du monde perçu lors de sa prise en charge par le discours. Les formes d'adéquation, labiles et culturellement façonnées par l'usage, entre ces deux sémiotiques - celle $\mathrm{du}$ monde naturel et celle des manifestations discursives des langages naturels -, font l'objet de la sémiotique figurative. (Bertrand 1993 : 190)

En effet, il essaie de clarifier la définition donnée par Greimas et Courtés. Selon lui, tous les systèmes de signes, verbaux ou non, nous présentent une relation entre la forme et un contenu. Cette relation contribue à la sémiose. Comme nous le voyons, ces définitions sont d'ordre structural. Quant aux proverbes, on s'aperçoit de leur spécificité comme un argument, d'une généralité qui doit coïncider le plus exactement possible avec l'expérience la plus large, pour être reçu comme «l'énoncé de la mémoire collective la moins limitée dans l'espace et dans le temps [qui repose] sur une opposition fondatrice d'un système de valeurs.» (Ollier 1976: 340). En effet, l'énoncé proverbial s'appuie sur une description (Une hirondelle ne fait pas le printemps) ou sur une narration (Qui vole un œuf vole un bouf) qui fixe le cadre de l'action.

Le monde naturel ou le monde visible décrit par les sémioticiens est comme un langage qui est composé d'un plan de l'expression et d'un plan du contenu. C'est la reformulation de Louis Hjelmslev qui est issue de la dichotomie saussurienne signifiant / signifié. La dichotomie hjelmslévienne permet de circonscrire l'espace de la sémantique. On doit pouvoir décrire la forme du contenu. C'est «l'articulation du contenu [qui] répond à la grille de lecture du monde sensible, soumise au relativisme culturel, qui constitue la substance du contenu.» (Bertrand 2000 : 105). Pour décrire ce contenu, nous pouvons effectuer une analyse lexicale qui tente de rendre compte des points de vue.

\section{Proposition d'une méthode d'analyse d'un corpus proverbial}

11 Nous utilisons l'hypothèse de la SPV selon laquelle les mots de la langue renvoient à des idéologies cristallisées dans les mots. Cette hypothèse a pour base la théorie de l'« Argumentation dans la Langue ».

Lorsque E est présenté comme argument pour C, le locuteur de E s'appuie sur un Garant. Quand on dit : " Il fait beau, allons nous promener », on s'appuie sur un garant qui établit la relation entre les deux énoncés (promenade $\rightarrow$ beau temps). Ainsi, dans le cadre 
théorique de l'Argumentation dans la Langue, si d'un énoncé $E_{1}$, on peut conclure à un énoncé $E_{2}$, c'est par le biais d'un troisième terme qui permet d'opérer ce lien conclusif entre $E_{1}$ et $E_{2}$. Autrement dit, c'est un " garant » qui autorise le passage de A à C. La classe des garants est appelée les «topoi » qui permettent d'opérer un choix parmi les chemins. En effet le «topos » devient le concept central dans la deuxième étape de la théorie de l'« Argumentation dans la Langue ».

Raccah détermine bien clairement les caractères principaux des topoi. Selon lui, l'orientation argumentative d'un énoncé est obtenue :

[...] par l'application, à certains éléments de la signification de la phrase, d'une règle d'inférence graduelle, que le locuteur présente comme : générale, en ce sens qu'elle est censée s'appliquer aussi à d'autres situations que celle qui fait l'objet de l'énoncé, et partagée, en ce sens qu'elle est supposée admise par l'ensemble des interlocuteurs.

Les règles de ce type ont été appelées ainsi, topoi (au singulier topos), en hommage à Aristote ; il s'agit cependant ici de concepts techniques ne renvoyant plus aux lieux d'Aristote (sauf pour leurs connotations). (Raccah 1990 : 182)

Les mouvements argumentatifs s'appuient donc sur ces " règles d'inférence graduelles ", des topoi dont dépend l'orientation argumentative de l'énoncé. Ils sont des principes généraux, qui servent d'appui au raisonnement, mais ne sont pas le raisonnement. Raccah caractérise les topoi de façon suivante :

Si l'énoncé $\mathrm{E}$ indique qu'une entité $\mathrm{X}$ possède une certaine propriété quelconque $\mathrm{P}_{1}$ et si ce fait permet (au locuteur) d'évaluer $\mathrm{X}$ dans une échelle $\mathrm{P}$, appelée champ topique de l'énoncé $\mathrm{E}$, les topoi applicables à $\mathrm{E}$ ont l'une des formes suivantes :

// plus $x$ est $P$, plus y est $Q / /$

// plus $x$ est $P$, moins y est $Q / /$

$/ /$ moins $x$ est $P$, plus y est $Q / /$

// moins x est $P$, moins y est $Q / /$ ». (Raccah $1990: 182)$

En somme, nous avons les formules suivantes :

plus $P$, plus $Q$

moins $P$, moins $Q$

plus $P$, moins $Q$

moins $P$, plus $Q$

ou bien en une seule formule :

// plus (ou moins) P, plus (ou moins) Q //

C'est « la situation d'énonciation qui détermine la validation de certains topoi en fonction du "bagage culturel" et des croyances de locuteur ». (Raccah $1990: 184)$

Cela montre que, non seulement une connaissance linguistique est nécessaire, mais des préoccupations cognitives sont aussi indispensables. Nous savons que la langue, qui est " un produit de l'esprit humain », « est en quelque sorte, un miroir des représentations cognitives des locuteurs » (Raccah $1990: 186)$. Ce qui est intéressant, c'est que les « topoi reflètent les croyances et les intentions des locuteurs et ne peuvent pas être déterminés par la seule analyse de la phrase ». C'est pourquoi, affirme-t-il : les « topoi qui font le lien entre les connaissances linguistiques et les connaissances du monde, font partie du "bagage cognitif" des locuteurs» (Raccah 1990 : 182). Par exemple: Il fait chaud, allons nous promener ", la conclusion montre que l'on a accepté le topos // plus il fait chaud, plus la promenade est agréable //, mais ce topos ne se valide pas dans les pays chauds. Pour ces pays, cet énoncé est un énoncé paradoxal (Bruxelles et alii 1993 : 9), car le point de vue imposé par la chaleur est différent du point de vue de l'énoncé «allons nous promener »: 
dans les pays chauds, on accepte le topos // plus il fait chaud dehors, plus on est à l'aise chez soi //.

De ce fait, nous devons admettre que «l'étude systématique des topoi ne relève pas de la linguistique mais d'une anthropologie culturelle (Bruxelles et alii 1993 : 2). En effet, le topos est issu d'une certaine idéologie et pourrait tout aussi bien être remplacé par un topos différent qui est engendré par une autre idéologie. Ce sont les points de vue qui évoquent ces idéologies et qui viennent de la culture. Les topoi montrent l'intersubjectivité entre les personnes parce que, comme nous avons dit au début, le topos est « une catégorie de garants d'argumentation [et ces garants], la rhétorique l'a montré, sont présentés par les locuteurs comme généraux et partagés par l'ensemble de la communauté linguistique (intersubjectivité) » (Raccah 2005a : 186).

Dans les topoi, les deux entités qui sont reliées, sont appelées les « champs topiques », ou plus précisément, un topos est un couple de « champs topiques ». " Un champ topique est, en gros, "une façon de voir" une entité, une propriété, une relation. Cette façon de voir est, elle-même, déterminée par la façon dont on voit une autre entité, une autre propriété ou une autre relation : c'est-à-dire par un autre champ topique »(Raccah 1990 : 195). En effet, « un champ topique contient une gradation qui dépend d'une valuation implicite dans les énoncés. Cette gradation correspond à une manière de voir un certain domaine de la réalité, domaine que [Raccah appelle] champ conceptuel » (Bruxelles et alii 1993 : 5). Par conséquent, le champ conceptuel est organisé par un jugement de valeur. Le champ topique se représente donc comme un couple dont le premier terme est un «champ conceptuel » et le deuxième terme est un "principe de gradation de ce champ ». Ce deuxième terme est ou bien une « évaluation du genre bien / mal », ou bien lui-même un « champ topique» qui peut servir de gradation pour un autre champ topique. Raccah formule cette définition de la façon suivante :

Le couple $(\mathrm{X}, \mathrm{Y})$ est un champ topique si et seulement si l'une des deux conclusions suivantes est respectée : I) X est un champ conceptuel et $\mathrm{Y}$ est une valeur (bien ou mal) Ou bien II) $\mathrm{X}$ est un champ conceptuel et $\mathrm{Y}$ est un champ topique. (Bruxelles et alii $1993: 6)$

Il affirme également :

Un champ topique peut être représenté par une chaîne de champs topiques emboîtés les uns dans les autres, de telle sorte que chaque champ topique est caractérisé d'une part, par un champ conceptuel (l'entité, la propriété ou la relation), et d'autre part, par le champ topique qu'il contient, lequel est lui-même caractérisé par un champ conceptuel et par le champ topique qu'il contient, et ainsi de suite jusqu'à un champ topique élémentaire. Ce dernier étant un principe de valuation, introduit une gradation dans le champ topique qui le contient. (Raccah 1990 : 195)

Apparemment, les champs topiques semblent être circulaires mais ils ne le sont pas car il y a toujours un jugement de valeur final qui donne un coup d'arrêt à la circularité. Cette caractéristique est nommée la "récursivité » des champs topiques. Ainsi, à partir de champs topiques élémentaires, nous pouvons construire des chaînes "croissantes " de champs topiques. Chaque champ topique élémentaire (il faut signaler que les champs topiques élémentaires sont le dernier maillon d'une chaîne définitoire) contient une évaluation qui peut agir sur tous les champs topiques qu'il contient. C'est pourquoi, on peut considérer le champ topique en même temps comme « une façon de voir » un champ conceptuel, et comme "un principe de gradation» pour d'autres champs topiques. Raccah donne ensuite les remarques suivantes: 
À chaque champ topique $\mathrm{CT}$ correspond un topos, qui lui est canoniquement associé : il s'agit du couple ( $\left.\mathrm{CT}, \mathrm{CT}^{\prime}\right)$, où $\mathrm{CT}^{\prime}$ est le premier champ topique enchâssé dans CT.

À chaque mot est associé un ou plusieurs champs topiques (s'il y en a plusieurs, le mot est argumentativement ambigu, phénomène qui se produit assez souvent, même dans le cas de mots informativement non-ambigus). Un champ topique associé à un mot est dit intrinsèque à ce mot. Un topos intrinsèque à un mot est le topos canoniquement associé à un champ topique intrinsèque à ce mot. (Raccah $1990: 196)$

23 Cette deuxième remarque est pertinente pour notre travail concernant la description des proverbes, et qui peut être semblable aux notions de sème et de sémème des sémioticiens. Leur différence consiste cependant en ce que les sèmes sont des traits distinctifs relatifs au signifié alors que les topoi sont d'ordre discursif relatif aux énoncés. Puisque les topoi se présentent dès le niveau lexical, le modèle topique est celui qui permet de choisir les descriptions à associer aux mots des proverbes.

Ajoutons que la signification d'une unité lexicale est un «faisceau de topoi ", à savoir l'ensemble des topoi dont cette unité autorise l'application et le sens du proverbe dépend de cette signification, car l'occurrence d'une unité lexicale autorise l'application d'un topos. Dans ces conditions, l'occurrence par exemple d'un mot-de-langue ne renvoie pas à un objet, mais induit une certaine vision de la situation. Ce sont ces points de vue qui nous aident à comprendre le sens des proverbes. La théorie des topoi se propose donc d'évacuer toute référence à ce qui est objectif et à ce qui est informatif. En ce qui concerne les proverbes, ce sont en effet les points de vue cristallisés dans les mots qui sont pertinents car liés à la vision d'une communauté linguistique.

La langue choisit certains topoi pour les «cristalliser» dans ses mots, sous forme de champs topiques. Ainsi les champs topiques lexicaux associés à la description des mots permettent de rendre compte des points de vue que ces mots provoquent. En d'autres termes, « les points de vue que les mots imposent s'expriment par des champs topiques lexicaux, qui permettent de décrire un point de vue sur une entité au moyen de points de vue d'autres entités " (Raccah 2005b : 228). Les topoi lexicaux sont dans la langue et ils peuvent montrer les points de vue cristallisés dans le lexique, c'est-à-dire les mots-delangue. Mais il y a d'autres points de vue qui apparaissent dans certaines situations par les énoncés. Raccah appelle les topoi évoqués par les énoncés « les topoi dynamiques ». Il faut souligner qu'il y a un rapport entre l'« énoncé » et « le point de vue » et il définit trois types d'énoncés, « doxaux », « a-doxaux » et " paradoxaux ».

Les énoncés doxaux sont les énoncés dans lesquels « le topos dynamique est exactement celui qui apparaît dans la description de la phrase » (Bruxelles et alii 1993 : 9). Quand dans un énoncé, les topoi lexicaux des mots donnent des points de vue contraires, l'énoncé devient "paradoxal ». Les énoncés «a-doxaux» sont ceux dans lesquels, le "topos dynamique » ne se contente pas de reprendre un constituant topique de la phrase, «le topos lexical », mais « il le combine à d'autres principes argumentatifs, éventuellement extra-linguistiques » (Bruxelles et alii 1993 : 9).

Après avoir présenté les principes fondamentaux de la théorie de la SPV, nous passons à la description des proverbes pour voir si le modèle topique, qui peut capter les points de vue liés aux mots, peut être suffisant pour rendre compte de la dimension figurative des proverbes. 


\section{La description de certaines propriétés de proverbes}

Nous allons analyser certains proverbes persans pour montrer que la dimension figurative des proverbes pourrait être décrite par les topoi lexicaux et dynamiques du point de vue du plan du contenu.

Signalons que l'outil formel que nous utilisons, à savoir la théorie des topoi, constitue un modèle pour décrire aussi bien le sens de l'énoncé que la signification du lexique. Car selon Raccah :

la langue est un produit de l'esprit humain et [...] ses structures sont des manifestations de structures plus abstraites de cet esprit, structures dont d'autres manifestations, distinctes des premières mais néanmoins analogues, se retrouvent dans l'usage de la langue et, plus généralement, dans la manière dont l'esprit gère les connaissances. Ainsi, les structures de l'expression linguistique (considérée comme un produit, et non comme une production) sont la trace, dans le domaine de la langue, de structures plus abstraites (appelons-les cognitives)». (Raccah 1999 : 20) [...] la langue est, en quelque sorte, un miroir des représentations cognitives des locuteurs ». (Raccah 1999 : 53)

Ainsi, semblera-t-il évident que la cognition humaine, considérée dans sa spécificité, est fondamentalement liée au langage et à la langue et que ce dernier a joué et continue de jouer un rôle de premier plan dans la conceptualisation humaine. Parmi les éléments de la langue, le lexique est associé directement à la base de connaissances. Et pour l'étudier, nous avons à notre disposition le modèle topique. En effet «les topoi permettent de "capturer", de manière directement utilisable, les connaissances concernant les variations de paramètres (objectifs ou subjectifs) » (Raccah 1999 : 55-56).

En ce qui concerne les proverbes, dans la plupart des cas, ce sont des énoncés $a$-doxaux parce qu'ils ajoutent d'autres points de vue aux mots, et ces façons de voir sont évoquées par la dimension figurative. Nous avons déjà montré comment la figurativité qualifie tout contenu de la langue qui a le rapport avec le monde naturel et la réalité perceptible.

Considérons le proverbe persan suivant :

(1) J'aime la courroie de ton sac, tant que tu as de l'argent dans ton sac, je suis ton

ami [Gorboone bande kifetam, ta pool dari refigetam]. (Shahri 2005)

Nous avons un système de représentation en même temps verbale et visuelle qui se trouve corrélé à une figure signifiante du monde perçu. C'est une observation qui fait écho à une expérience déjà acquise. Il nous figure une image du monde naturel pour nous suggérer le sens «Je t'apprécie pour ton argent »! En effet, le proverbe nous présente une façon de voir, ou mieux dire une vision qui est déjà habitée par un sens « qui lui donne une fonction dans le spectacle du monde » (Merleau-Ponty 1945 : 64). Ce monde du sens commun se déploie comme un langage figuratif articulé en "propriétés sensibles" inséparables de "propriétés discursives ». "Ces propriétés peuvent être formulées dans les termes d'une organisation narrative qui sous-tend la perception de chaque figure du monde nature : une micro-syntaxe, réglant les interactions entre les sujets percevants et les objets perçus » (Bertrand 2000 : 101). Ce sont ces mêmes figures, déployées au sein de parcours figuratif, qui constituent la dimension figurative des discours, grâce à elles, le «monde nous parle ». Parmi les différents discours, les proverbes, comme celui indiqué ci-dessus, jouent avec les distorsions de cette figurativité dans le langage. 
la sémantique : la description de la substance du contenu. C'est la saisie du contenu. Nous pouvons élargir aussi le champ topique de ce proverbe, par le topos culturel : // plus on a du pouvoir, plus on a des amis // pour affirmer que «l'articulation du contenu répond à la grille de lecture du monde sensible, soumise au relativisme culturel, qui constitue la substance du contenu » (Bertrand 2000 : 105). L'amitié est liée au pouvoir qui est imposé par l'argent. C'est une façon de voir le monde que le proverbe montre par l'emploi des mots sac, argent, ami. Les points de vue sont lexicalisés dans les champs lexicaux. Ainsi le proverbe nous suggère-t-il le topos dynamique : l'argent favorise l'amitié.

(2) Une fleur ne fait pas le printemps [Bâ yek gol, Bahar nemisheh]. (Salahshoor 2008)

Ce proverbe persan n'est non plus un commentaire sur le printemps ni sur la fleur, il est en tout point identique à la phrase suivante. «Avec une fleur, on ne peut pas parler du printemps ». Cela nous suggère une façon de voir. Ce proverbe fait voir et fait croire qu' " une seule chose qui est bonne n'est pas suffisante pour atteindre un résultat favorable ». Ce qui est important, c'est la corrélation entre la « fleur » et le « printemps ». Cette façon de voir et ce point de vue positif cristallisé dans le mot vient du monde naturel (le langage figuratif).

Concernant la dimension figurative de ce proverbe, signalons encore que pour MerleauPonty, voir, c'est comprendre et interpréter des rapports de sens. Comme l'écrit Michel de Certeau dans un commentaire du Visible et l'invisible de Merleau-Ponty : "Voir est déjà un acte de langage. Cet acte fait des choses vues l'énonciation de l'invisible texture qui les 
noue » (De Certeau 1982 : 97). Mais du point de vue de la sémantique, cette façon de voir est déjà dans les mots « fleur » et « printemps ». Nous pouvons avoir le topos suivant :

$\mathrm{T}: / /$ plus des fleurs, plus le printemps //, // moins des fleurs, moins le printemps

$/ /$ apporte les meilleures choses » et les connotations : « la promesse et l'espoir de la vie, le début d'une chose, le début d'une joie, etc. » (Dehxoda 2007). Et pour les connotations de la fleur, dans les dictionnaires persans, est apporté «le résultat, le profit, l'intérêt " (Dehxoda 2007). Ces connotations sont importantes pour créer le sens. Elles sont comme un «fragment d'idéologie » selon Roland Barthes. Pour lui, la connotation permet de rattacher un énoncé pris en synchronie à une double diachronie, qui est d'une part celle de la langue et d'autre part celle du processus socio-culturel. C'est un lien rattachant la parole à l'écriture et à l'Histoire (Barthes 1985 : 78). Nous nous approchons ainsi de l'idée de Raccah, selon laquelle les mots des langues imposent les idéologies, c'est-à-dire les points de vue qu'il est nécessaire d'adopter pour comprendre. Autrement dit, les mots de langue imposent des contraintes sur les points de vue. Signalons qu'en sémiotique, un système connotatif peut être l'un des systèmes créateurs du sens, mais il doit être complété par d'autres mécanismes. Le modèle topique peut être un modèle pour décrire les points de vue évoqués par ces fragments de l'idéologie qui sont dans les mots. C'est-àdire, la connotation peut créer le topos dynamique qui est plutôt le mariage du topos lexical et du topos culturel. Nous pouvons décrire la figurativité de façon suivante :

\begin{tabular}{|c|c|}
\hline Dimension figurative (la mimesis) & Eléments de SPV \\
\hline forme de l'expression 晦 Mot & 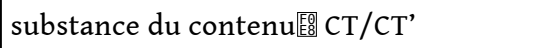 \\
\hline Une micro-syntaxe & mini-programme argumentatif \\
\hline $\begin{array}{l}\text { Fleur } \\
\text { Printemps }\end{array}$ & $\begin{array}{l}\text { <AVOIR DES PROFITS, bien> } \\
\text { <AVOIR DES PROFITS, } \\
\text { <ATTEINDRE UN BON RESULTAT, bien>> }\end{array}$ \\
\hline
\end{tabular}

Pour le proverbe ci-dessus, nous pouvons avoir le topos culturel: // plus c'est le printemps, plus on a la promesse de la vie et on a de la joie //. Pour avoir enfin le topos dynamique du proverbe : // plus de bonnes choses, plus le résultat positif et efficace //.

(3) Si tu as de la patience, tu pourras faire du helva avec du raisin cru [Gar sabr koni, ze gooreh halva sazi]. (Shahri 2005) Ce proverbe veut dire: "plus on a de la patience, plus on peut changer de mauvaises situations en bonnes situations ». La corrélation entre les mots: la patience [sabr], le helva [halva] et le raisin cru [ghooreh] est importante pour suggérer le sens du proverbe. Helva est une sorte de gâteau sucré et le raisin cru [ghooreh] est le raisin qui n'est pas mûr et qui est acide (Dehxoda 2007). 
Cela donne une façon de voir qui existe déjà dans la dimension figurative de ce proverbe. Nous pouvons avoir le topos culturel suivant: // plus de patience, plus de bonnes choses $1 /$.

Le champ topique lexical de patience est :

$\mathrm{CT}$ : < Mettre plus de temps <Avoir de bonnes choses, bien>, <Avoir de bonnes choses, bien>>.

Le terme "helva» a pour connotation toute chose douce et agréable et le raisin cru connote non seulement tout fruit cru et acidulé, mais encore un enfant inexpérimenté. Ces connotations sont en rapport avec la patience qui rend les choses plus agréables. Il faut savoir attendre pour avoir de bonnes choses :

\begin{tabular}{|c|c|}
\hline Dimension figurative (la mimesis) & Eléments de SPV \\
\hline forme de l'expression 酒 Mot & 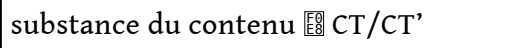 \\
\hline Une micro-syntaxe & mini-programme argumentatif \\
\hline Patience & <METTRE PLUS DE TEMPS, bien> \\
\hline Helva & <AVOIR DE BONNES CHOSES, bien> \\
\hline Raisin cru & $<$ AVOIR DE MAUVAISES CHOSES, mal> \\
\hline
\end{tabular}

4 Le topos dynamique de ce proverbe est: // plus on patiente, plus on a de bonnes choses $1 /$.

Ajoutons que nous avons aussi une expérience temporelle. Quand on a de la patience, c'est dire que l'on se donne plus de temps pour avoir un bon résultat. Cette notion de temps existe dans beaucoup de proverbes avec des images différentes, en effet, la forme de l'expression varie. Par exemple :

(4) « Les gouttent se rassemblent, le résultat c'est la mer » [Gatreh gatreh jam gardad, vangahi darya shavad]. (Salahshoor 2008)

Dans cet énoncé proverbial, c'est l'aspect pictural du temps qui est évoqué par les mots : " goutte » [gatreh] et « mer » [Darya] qui évoquent également la patience. " Goutte » [gatreh ] connote une chose très petite, une quantité très petite ; en revanche la «mer» [darya] nous suggère une grande quantité, une immensité. Nous pouvons avoir le topos : // plus de gouttes, plus la possibilité d'avoir la mer // ou plus encore : // plus de petites choses, plus la possibilité d'avoir une quantité considérable //. L'opposition goutte / mer met en scène une dimension figurative que l'on peut décrire de manière ci-dessous :

\begin{tabular}{|c|c|}
\hline Dimension figurative (la mimesis) & Eléments de SPV \\
\hline forme de l'expression 㯺 Mot & 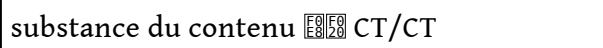 \\
\hline Une micro-syntaxe & mini-programme argumentatif \\
\hline Goutte & <AVOIR DES PETITES CHOSES, bien> \\
\hline Mer & AVOIR UNE QUANTITE CONSIDERABLE, bien> \\
\hline
\end{tabular}


(5) Où tu vas, le ciel a la même couleur. [Har jâ ravi, Aseman hamin rang ast].

(Salahshoor 2008) verbe «aller» [raftan], l'autre, la stabilité «la couleur du ciel» [rangue aseman]. La corrélation entre le verbe « aller » et « la couleur du ciel » est important pour suggérer le sens du proverbe " partout il y a des problèmes », « on ne peut trouver un lieu utopique ». Pour suggérer le sens, le proverbe nous donne une «façon de voir»: déplacement / stabilité des caractères. Comme tous les proverbes, il s'agit de typifier une situation de manière figurative. Pour décrire les effets de sens figuratif, nous allons évoquer l'autonomie de la langue par des champs topiques. C'est une reformulation, comme dans d'autres exemples, des points de vue cristallisés dans les mots. L'apport de la formulation raccahienne consiste à faire apparaître « la substance » des mots :

\begin{tabular}{|c|c|}
\hline Dimension figurative (la mimesis) & Eléments de SPV \\
\hline forme de l'expression 醌 Mot & 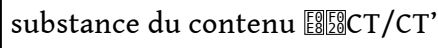 \\
\hline Une micro-syntaxe & mini-programme argumentatif \\
\hline Aller & $<$ SE DEPLACER, bien> \\
\hline Couleur du ciel & $<$ AVOIR LA MEME CHOSE, mal> \\
\hline
\end{tabular}

Nous pouvons le montrer par le topos dynamique :

// plus on se déplace, plus on découvre les mêmes choses, les mêmes problèmes //.

Ces quelques observations avaient pour but de décrire la figurativité des énoncés proverbiaux par la SPV.

\section{Pour conclure...}

Il faut dire que selon la théorie de l'AdL, la langue est fondamentalement un moyen d'interaction. Elle n'est un moyen d'information que de façon dérivée ou seconde. En effet, la langue se révèle " un instrument d'action » au moins et plus qu'un instrument d'information. Un aspect essentiel de la langue est en particulier l'argumentation. En considérant cette théorie et en nous appuyant sur l'idée de Raccah, selon laquelle les mots des langues imposent les idéologies, c'est-à-dire les points de vue qu'il est nécessaire d'adopter pour comprendre le sens des énoncés, nous avons pu analyser la dimension figurative de certains proverbes. Le proverbe ne pourrait être analysé au moyen des concepts classiques de la linguistique. En effet, ils sont inaptes à rendre compte de la spécificité de l'énoncé proverbial. Nous croyons en fait que les proverbes illustrent les points de vue lexicalisés dans les mots. L'utilisation des concepts comme champ conceptuel et champs topique nous offre l'avantage de décrire la dimension figurative de la manifestation du sens. 


\section{BIBLIOGRAPHIE}

Barthes Roland, L'aventure sémiologique, Paris, Seuil, 1985.

Bertrand Denis, Précis de sémiotique littéraire, Paris, Nathan, 2000.

Bruxelles Sylvie, Ducrot Oswald et Raccah Pierre-Yves, « Argumentation et champs topiques lexicaux ", Cahiers de Praxématique, n 21, 1993, pp. 88-104.

De Certeau Michel, « La folie de la vision », Esprit, nº66, 1982, pp. 89-99.

Dehxoda Ali Akbar, Dictionnaire de la langue persane, Téhéran, Université de Téhéran, 2007.

Greimas Algirdas Julien, «Idiotismes, proverbes, dictons » Cahiers de lexicologie, $\mathrm{n}^{\circ}$ 2, 1961, pp. 41-61.

Greimas Algirdas Julien et Courtés Joseph, Sémiotique. Dictionnaire raisonné de la théorie du langage, Paris, Hachette, 1986.

Kleiber Georges, «Sur le sens des proverbes », Langages, $n^{\circ} 139,1999$, pp. 39-58.

Kleiber Georges, « Y a-t-il de la métaphore sous les noms propres en antonomase ? ", RASK, $\mathrm{n}^{\circ} 2$, 1994, pp. 3-24.

Merleau-Ponty Maurice, Phénoménologie de la perception, Paris, Gallimard, 1945.

Ollier Marie-Louise, « Proverbe et sentence, le discours d'autorité chez Chrétien de Troyes », Revue des sciences humaines, juillet-septembre, $\mathrm{n}^{\circ}$ 163, t. XLI, 1976, pp. 329-357.

Raccah Pierre-Yves, « Signification, sens et connaissance : une approche topique », Cahiers de linguistique française, $\mathrm{n}^{\circ} 11,1990$, pp. 179-198.

Raccah Pierre-Yves, « Une description de l'excessivité en sémantique des points de vue », Travaux de linguistique du CERLICO, $\mathrm{n}^{\circ}$ 18, 2005a, pp. 171-190.

Raccah Pierre-Yves, «Une sémantique du point de vue : de l'intersubjectivité à l'adhésion », in «L'Énonciation identitaire : entre l'individuel et le collectif », dir. par Danielle Forget, Discours social, vol. XXI, 2005b, pp. 205-242.

Rodegem François, « La parole proverbiale », in Suard François et Buridant Claude (eds), Richesse du proverbe, Actes du colloque de parémiologie [Lille, 6-8 mars 1981], Lille, vol. I, Typologie et fonctions, 1984, pp. 121-135.

Salahshoor Soheila, Les meilleurs proverbes iraniens, Téhéran, Arvand, 2008.

Shahri Ja'far, Le sucre et le sel, les proverbes téhéranais, Téhéran, Moïn, 2005.

Visetti Yves-Marie et Cadiot Yves, Motifs et proverbes. Essai de sémantique proverbiale, Paris, PUF, 2006.

\section{RÉSUMÉS}

Les proverbes ont une valeur culturelle et ils nous montrent les manières de penser d'une société donnée. Pour décrire de manière adéquate ce système de valeurs liées aux conditions sociales 
dans lesquelles la langue est actualisée, nous avons recous à la Sémantique des Points de Vue et à la sémiotique. Notre hypothèse est fondée sur l'étude approfondie des points de vue lexicalisés dans les proverbes qui correspondent par ailleurs à la «dimension figurative » du discours définie par les sémioticiens. En effet, l'objectif de cette recherche est de mettre en évidence que les proverbes par leur figurativité nous imposent une «façon de voir » qui est lexicalisée dans les mots et nous pouvons les décrire en termes de champs topiques définis par Pierre-Yves Raccah.

The proverbs have a cultural value and they show the way of thinking of a society. To give a description about this system of values related to the social conditions in which a language is actualized, we apply the semantics of the points of view and the semiotics. Our basic hypothesis is founded on the study of the points of view lexicalized in the proverbs that corresponded to the "figurative dimension" of the discourse defined by the semioticians. In fact, the objective of this research is to bring out that the proverbs impose to us a "manner of see" by their figuratively. This "manner" is lexicalized in the words and we can describe by "topique fields" defined by Pierre-Yves Raccah.

INDEX

Mots-clés : proverbe, dimension figurative, topoi, sémantique des points de vue, sémiotique

Keywords : proverb, figurative dimension, topoi, semantics of points of view, semiotics

\section{AUTEUR}

\section{MARZIEH ATHARI NIKAZM}

Maître-assistante à l'Université Shahid Beheshti de Téhéran, Iran 